\title{
Use of Abamectin as An Eco-Friendly Pesticide Against Diamondback Moth on Cabbage Crop
}

\author{
M. M. Morsy ${ }^{1 *}$, Adel A. Elwan ${ }^{2}$ \\ 1Plant Protection Department, Faculty of Agriculture, Zagazig University, Zagazig, Egypt \\ 2Pedology Department, Desert Research Center, Cairo, 11753, Egypt. \\ *Corresponding author: Tel.: +201011824240, E-mail: mariam.mosaad@yahoo. com \\ DOI: 10.21608/jalexu.2022.111943.1029
}

\section{Article Information}

Received:November $25^{\text {th }}$ 2021

Revised: December $27^{\text {th }}$ 2021

Accepted:December $29^{\text {th }}$ 2021

Published: December $31^{\text {st }}$ 2021
ABSTRACT: The diamondback moth, Plutella xylostella, is the greatest damaging insect of cabbage plants. Two field experiments were conducted at private farm in El-Kattawia area, Abu-Hammad City, Sharkia Governorate, Egypt to assess the effectiveness of abamectin 1.8 EC against cabbage diamondback moth. Two field experiments were performed at the same farm in different seasons (2019 and 2020). The experiments were conducted in a randomized block design with a plot size of $4 \times 5 \mathrm{~m}$ with three replications. The concerned treatments were enjoined four times at 14 days intervals beginning from the $30^{\text {th }}$ day after cultivation. The pneumatic Knapsack sprayer was used to spray fluid (600 liters per hectare). Observations on larval population were made before spraying and on 3, 7, 10, and 14 days after spraying from 10 randomly tagged plants in each plot. Four doses of abamectin @ 9, 11,13, and $15 \mathrm{~g}$ a.i.ha ${ }^{-1}$ were assessed against the Plutella xylostella under study. Besides, Cypermethrin 10 EC @ $70 \mathrm{~g}$ a.i./ha, endosulfan 35 EC @ $420 \mathrm{~g}$ a.i./ha, spinosad 45 SC @ $75 \mathrm{~g}$ a.i./ha, and an untreated blank were also included in the field experiment.The findings concluded that treating with abamectin at $15 \mathrm{~g}$ a.i./ ha was the highest mean reduction which meaningfully blocked the population of diamondback moth larvae and recorded a mean reduction of 72.5, 70.7, 75.2, and 78.0 percent from untreated check after first, second, third and fourth sprays, respectively. While the results obtained from abamectin at $11 \mathrm{~g}$ a.i./ ha were on par with standard check spinosad at $75 \mathrm{~g}$ a.i./ha and excelled over cypermethrin and endosulfan. A similar trend was also observed in the second experiment. The yield of cabbage heads also increased significantly at all the doses tested in the two experiments under field conditions as compared to the control plot. The study demonstrates the potentiality of abamectin $1.8 \mathrm{EC}$ as an eco-friendly bioinsecticide against the diamondback moth (Plutella xylostella). The doses of the studied abamectin@ @ 15,13, and $11 \mathrm{~g}$ a.i.ha ${ }^{-1}$ were found to be highly effective to control the infestation of diamondback moth in the cabbage plants under the conditions of a semi-arid zone in Egypt.

Keywords: Eco-friendy, Abamectin, Diamondback moth, Cabbage, Egypt.

\begin{abstract}
INTRODUCTION
The cabbage plant is an important vegetable crop from the cruciferous group and is widely grown all over Egypt. It is mainly used either as a cooked vegetable or as a raw salad in households and hotels. Among the various insect pests attacking cabbage, diamondback moth (DBM), Plutella xylostella L., is the most dreaded pest throughout the world and the annual cost incurred for managing this pest is estimated to be 1 billion USD (Talekar, 1990). A female moth deposits an average of 150 eggs over about 10 days (Capinera, 2018) DBM Plutella xylostella (Linn.), a butterfly of the family Plutellidae, is injurious to cruciferous plants. Where cabbages are a major food crop and insecticides are inefficient for control (Diab, 2011). The DBM incidence and damage of DBM are now found to be the most devastating one in Cole crops causing a 52 percent loss in marketable produce and thus assuming the status of national importance (Krishnakumar et al., 1986).
\end{abstract}

Extreme use of insecticides leads to resistance difficulties to these pesticides either individual or multiple (Dara 2020). In the 1990s, the use of synthetic insecticides has eliminated the application of natural insecticides. Nowadays, this insect pest resisted major insecticides viz, BHC and DDT (Dara 2020). Numerous recent pesticides were suggested to manage Plutella xylostella L. but the observation showed that $60 \%$ of the farmers cannot control this pest effectively (Harika et al., 2019). In the 1980s, there have several new pesticides were produced to reduce $P$. xylostella viz, triazophos, permethrin, fenvalerate, cartop, and methomyl. These insecticides were found to be less active after three to five years of foliar application by the agriculturalists (Fauziah et al., 2012).

Vegetable crops hold residues of a mixture of chemicals since these are sprayed at growth stages of the crop, causing health problems to the customers. As the elimination of chemical 
pesticides is unviable in the farmers, it has required the use of substitute eco-friendly insecticides for maintainable controlling of diamondback moth which can reduce it to minor pest status by the natural enemies and eco-friendly materials. The change of resistance against these outdated insecticides may be simply degraded by the new group of sustainable compounds to protect the environment (Harika et al., 2019). Keeping because of the seriousness of this pest and the economic importance of the cabbage crop, the current research was conducted to evaluate the effectiveness of the best-known insecticides viz., abamectin, spinosad, cypermethrin, and endosulfan under field conditions of a semi-arid zone at El-Kattawia, Abu-Hammad City, Sharkia Governorate, Egypt against Plutella xylostella on cabbage.

\section{MATERIALS AND METHODS}

\section{Study site and experiments layout}

The research work was done in a semiarid zone of Egypt to assess the effectiveness of abamectin 1.8 EC on the cabbage diamondback moth (Plutella xylostella $\mathrm{L}$ ). The two experiments were carried out on two seasons under field conditions. The field experiments were conducted in a private farm at El-Kattawia area, Abu-Hammad City, Sharkia
Governorate, Egypt during the 2019 and 2020 seasons (Fig. 1). The field experiment area is situated between latitudes $30^{\circ} 33^{\prime} 47^{\prime \prime}$ and $30^{\circ} 33^{\prime}$ $52^{\prime \prime} \mathrm{N}$; and longitude $31^{\circ} 39^{\prime} 41^{\prime \prime}$ and $31^{\circ} 39^{\prime} 46^{\prime \prime} \mathrm{E}$ (Fig. 1). In this region, the mean annual rainfall was $55 \mathrm{~mm}$ and the mean maximum and minimum temperatures were 31 and $22^{\circ} \mathrm{C}$, respectively (Egyptian Meteorological Authority, 2020). The soil texture, $\mathrm{pH}$, and soil salinity $(\mathrm{dS} / \mathrm{m})$ were determined using a 1:1 soil/water extract (Jackson, 1973). Soil organic carbon content was carried out using the Walkley-Black method (FAO, 1970) and available macronutrients ( $\mathrm{N}, \mathrm{P}$, and $\mathrm{K}$ ) were measured based on the standard methods outlined in Soil Survey Staff (2014). Soils of the farm fields have clay loam texture (moderately fine-textured soils), with $\mathrm{pH}$ at $7.9, \mathrm{EC}$ at $0.95 \mathrm{dS} / \mathrm{m}, 0.95 \%$ of organic carbon, $5.59 \mathrm{~g} \mathrm{~kg}^{-1}$ of total nitrogen, 7.27 $\mathrm{mg} \mathrm{kg}^{-1}$ of available phosphorus, and $105.13 \mathrm{mg}$ $\mathrm{kg}^{-1}$ of available potassium. The River Nile water was the source of the irrigation water. The farm ecosystem provides the cabbage plants with the optimum growth requirements (soil, water, and climate) to grow healthily. The experiments were performed in a randomized block design (RBD) with a plot size of $4 \times 5 \mathrm{~m}$ with three replications.

The treatments in each experiment were as follows:

\begin{tabular}{ccc}
\hline Treatments & Pesticides & Doses (g a.i./ha) \\
\hline $\mathbf{T}_{\mathbf{1}}$ & Abamectin1.8 EC & $\mathbf{9}$ \\
$\mathbf{T}_{\mathbf{2}}$ & Abamectin1.8 EC & $\mathbf{1 1}$ \\
$\mathbf{T}_{\mathbf{3}}$ & Abamectin 1.8 EC & $\mathbf{1 3}$ \\
$\mathbf{T}_{\mathbf{4}}$ & Abamectin 1.8 EC & $\mathbf{1 5}$ \\
$\mathbf{T}_{\mathbf{5}}$ & Spinosad 45 SC & $\mathbf{7 5}$ \\
$\mathbf{T}_{\mathbf{6}}$ & Cypermethrin 10 EC & $\mathbf{7 0}$ \\
$\mathbf{T}_{\mathbf{7}}$ & Endosulfan 35 EC & $\mathbf{4 2 0}$ \\
$\mathbf{T}_{\mathbf{8}}$ & Untreated check & -- \\
\hline
\end{tabular}




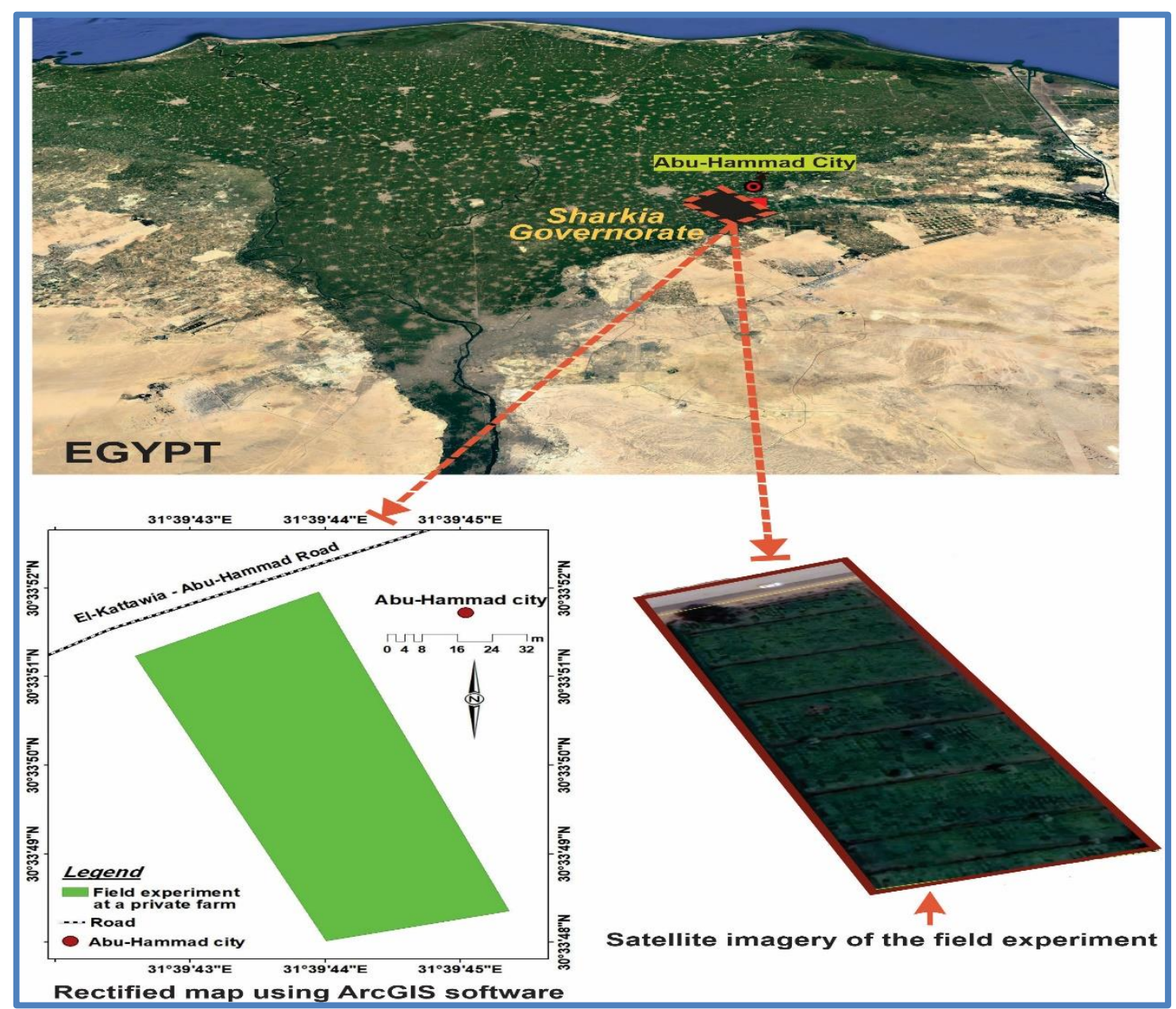

Fig. 1. Location of the field experiments at El-Kattawia area, Abu-Hammad City, Sharkia Governorate, Egypt

The treatments were imposed four times at 14 days intervals commencing from the $30^{\text {th }}$ day after planting with a pneumatic Knapsack sprayer using 600 liters of spray fluid per hectare. The third application was given 21 days after the second spray since the larval population did not cross the Economic Threshold Level ETL 14 days after the second spray in the experiment. Applications were done during morning hours in such a way to give uniform coverage on foliage and to avoid drift and photo-oxidation of the insecticides. Observations on larval population were made before spraying and on $3,7,10$, and 14 days after spraying from 10 randomly tagged plants in each plot. The cabbage yield was moreover documented throughout the harvest stage.

The analysis of variance was carried out by randomized block design using IRRISTAT ver 3.1. ANOVA was carried out for the field experiment. The percentages data were altered to arcsine percentage. The mean values of treatments were separated using Duncan's Multiple Range Test (DMRT) (Gomez and Gomez, 1976).

\section{RESULTS AND DISCUSSION}

Bioefficacy of the studied abamectin against Plutella xylostella L.

\section{a) First experiment (Season of 2019)}

The findings of the first field experiment of the 2019 season are outlined in Tables 1 and 2. The pretreatment population of DBM varied from 34.5 to 36.6 larvae per 10 cabbage plants. In the studied treatments, the highest value of larval reduction $(66.5 \%)$ was registered in treated plots at $15 \mathrm{~g}$ a.i.ha ${ }^{-1}$ of abamectin followed by $60.0 \%$ at $13 \mathrm{~g}$ a.i. $\mathrm{ha}^{-1}$ of abamectin and which was in line with cypermethrin 10 EC (58.7\%) on 3 days after treatments (DAT). While on 7 DAT, the doses of abamectin $15 \mathrm{~g}$ a.i.ha $\mathrm{h}^{-1}$ and $13 \mathrm{~g}$ a.i.ha ${ }^{-1}$ recorded $84.2 \%$ and $79.6 \%$ reduction in population, respectively, besides on 14 DAT was $64.1 \%$ and $59.2 \%$, respectively as shown in Table.1.

The buildup of the DBM population at 14 DAT necessitated the second spray. The population before treatment ranged between 23.8 and 65.5 per 10 plants. Abamectin $15 \mathrm{~g}$ a.i.ha ${ }^{1}$ registered an 82.4 percent reduction on 7 DAT and the lowest percent reduction was observed in 
endosulfan 35 EC (42.9\%). Though endosulfan and cypermethrin affected more reduction of DBM population till 3 DAT, all doses of abamectin observed more reduction compared to endosulfan and cypermethrin in the successive days. A similar trend was similarly observed in treated plots on 14 DAT. After the third spray, a significant reduction in the DBM population was observed in all the insecticide treatments. Among them, abamectin at 15 and $11 \mathrm{~g}$ a.i.ha ${ }^{-1}$ registered 89.2 and 73.5 percent reduction in population on 7 DAT whereas, the same results were obtained by spinosad 45 SC 75 g a.i.ha- ${ }^{-1}(73.5 \%)$ (Table 2). The same trend of efficacy was observed throughout the study period.

Seven days after the fourth round of spray, abamectin at $15 \mathrm{~g}$ a.i./ha documented a $91 \%$ reduction in larval population followed by a $13 \mathrm{~g}$ a.i.ha ${ }^{-1}$ of abamectin (84.1\%). On 10 DAT, abamectin at 9, 11, 13 and $15 \mathrm{~g}$ a.i.ha ${ }^{-1}$ caused 61.9, $67.5,71$, and $78.5 \%$ reduction, respectively, while endosulfan at $420 \mathrm{~g}$ a.i./ha and cypermethrin at 70 $\mathrm{g}$ a.i./ha listed $36.5 \%$ and $43.4 \%$ reduction, respectively. On $14 \mathrm{DAT}$, abamectin at $15 \mathrm{~g}$ a.i./ha itemized 67.9 percent reduction followed by abamectin at13 g a.i./ha(63.4\%) whereas, the standard checks, cypermethrin, and endosulfan recorded 34.9 and 24.0 percent reduction, respectively. A significant reduction in the DBM population was noticed in all the doses of abamectin, which were superior to the standard chemicals viz., cypermethrin 10 EC, endosulfan 35 EC, and spinosad $45 \mathrm{SC}$ as presented in Table 2.

\section{b) The second experiment (Season of 2020)}

The pretreatment population ranged from 52.5 to 57.1 larvae per ten plants. There was a significant reduction of DBM larval population after spraying insecticides. The population reductions were higher $(63.1 \%$ and $66.9 \%)$ in both of abamectin at $13 \mathrm{~g}$ a.i.ha ${ }^{-1}$ and $15 \mathrm{~g}$ a.i.ha ${ }^{-1}$ on 3 DAT, respectively. These findings agreed with cypermethrin $10 \mathrm{EC}(61.5 \%)$. While the foliar spraying of abamectin after 7 days (7 DAT) at 15 and $13 \mathrm{~g}$ a.i.ha ${ }^{-1}$ recognized reductions of $83.9 \%$ and $82.1 \%$, respectively. Whereas, abamectin at 15 $\mathrm{g}$ a.i. $\mathrm{ha}^{-1}$ verified a reduction of $62.1 \%$ against $57.6 \%$ using the dose of $13 \mathrm{~g}$ a.i.ha ${ }^{-1}$ on 14 DAT (Table 3).

After the second round of spray, abamectin at $15 \mathrm{~g}$ a.i.ha ${ }^{-1}$ registered a maximum of 84.5 percent reduction on 7 DAT and the lowest percent reduction was observed in endosulfan $35 \mathrm{EC}$ (44.8\%). Though endosulfan and cypermethrin recorded more percent reduction of DBM population up to $3 \mathrm{DAT}$, all tested doses of the abamectin increased more reduction compared to the tested doses from cypermethrin and endosulfan in the subsequent days. On 10 DAT, abamectin at $15 \mathrm{~g}$ a.i.ha ${ }^{-1}$ reduced to $71.3 \%$ and abamectin at 13 $\mathrm{g}$ a.i.ha ${ }^{-1}(67.6 \%)$. After the third round of spraying, abamectin at 15 and $11 \mathrm{~g}$ a.i.ha-1 registered 92.2 and 81.2 percent reduction in population on 7 DAT, respectively which was on par with spinosad at $75 \mathrm{~g}$ a.i.ha-1 $(80.9 \%)$. The same trend of effectiveness was recognized up to 14 days after treatments (Table 4). After the fourth round of spray, abamectin at $9,11,13$ and $15 \mathrm{~g}$ a.i.ha- 1 caused $62.1 \%, 70.2 \%, 77.1 \%$ and $86.0 \%$ decrease, respectively on 10 DAT. The doses of endosulfan at $420 \mathrm{~g}$ a.i.ha ${ }^{-1}$ and cypermethrin at 70 $\mathrm{g}$ a.i.ha $\mathrm{h}^{-1}$ noted reductions of $43.0 \%$ and $49.0 \%$, respectively. On 14 DAT, the doses of abamectin at $13 \mathrm{~g}$ a.i/ha and $15 \mathrm{~g}$ a.i $\mathrm{ha}^{-1}$ increased the reductions to $66.5 \%$ and $71.0 \%$, respectively. Whereas, endosulfan and cypermethrin reduced the population to $34.9 \%$ and $38.7 \%$, respectively. A major reduction of DBM population was detected in all the treatments of abamectin which was superior to the standard chemicals viz., spinosad 45 SC, cypermethrin $10 \mathrm{EC}$, and endosulfan 35 EC (Table 4).

The results of the present study conducted on bioefficacy of abamectin against cabbage diamondback moth, in the two experiments are depicted in Figs. 2 and 3. The results of the first experiment revealed that abamectin $15 \mathrm{~g}$ a.i./ ha registered the highest mean reduction $(72.5,70.7$, 75.2 , and 78 percent) of the DBM population from control after first, second, third, and fourth foliar sprayings, respectively. Likewise, abamectin at 11 g a.i./ ha recorded 61.5, 61.8, 63.4 and 66.7 percent, respectively. Abamectin showed a 50 percent reduction in larval population up to 14 DAT even at lower concentrations. Though the mortality was not higher immediately after treatment in abamectin, the damage was significantly reduced as compared to check. This may be due to the feeding of the larvae at different time intervals on treated plants which might have resulted in varying degrees of larval deformities due to reduced food consumption and loss of weight. In the second experiment, abamectin at 15 $\mathrm{g}$ a.i./ ha recorded $71.75,71.33,79.96$ and $80.63 \%$ mean reduction of the DBM population from control after first, second, third, and fourth foliar sprayings, respectively, respectively. The bioefficacy trend of abamectin against $P$. xylostella was similar in the two field experiments for all the doses. Abamectin at all concentrations and doses excelled when compared to cypermethrin and endosulfan. The treated plants with abamectin at $15 \mathrm{~g}$ a.i./ha have got a reduction in DBM population over control in the range of 84.5 to 92.5 percent in the second field experiment on 7 DAT. This finding showed that abamectin has a remarkable knockdown effect on the DBM larvae, which could be due to the innate nature of abamectin to interfere with chloride ion permeability and GABA receptors and which were well demonstrated by Scott and Dwe (1985). The 
present findings obtain strength from the report of Murugan and Ramachandran (2000) who reported that Vertimec ${ }^{\circledR} 1.8$ EC @ $15 \mathrm{~g}$ a.i. ha ${ }^{-1}$ recorded $0.25,0.43$, and 0.40 larva per plant on 5 days after first, second and third sprayings, respectively compared to untreated check which recorded 3.55, 3.33, and 2.65 larvae per plant, respectively. Sengonca and Liu (2001) also pinpointed that GCSC- BtA was highly toxic to the third instar of $P$. xylostella with $91.18 \%$ mortality followed by abamectin with $78.0 \%$. Agrimec $^{\circledR} 1.8$ EC on cabbage and cauliflower was the most effective insecticide in controlling DBM and was superior to spinosad, profenofos, lufenuron, and cyhalothrin (Syed et al., 2004). Though the mortality was not higher immediately after treatment in abamectin 1.8 EC, the destruction of the studied crop was significantly decreased as compared to the control. This may be due to the feeding of the larvae at various time intervals on treated plants which might have resulted in varying degrees of larval deformities due to reduced food consumption and loss of weight.

Table 1. The larval populations after the first and second rounds of application in the first field experiment (Season of 2019).

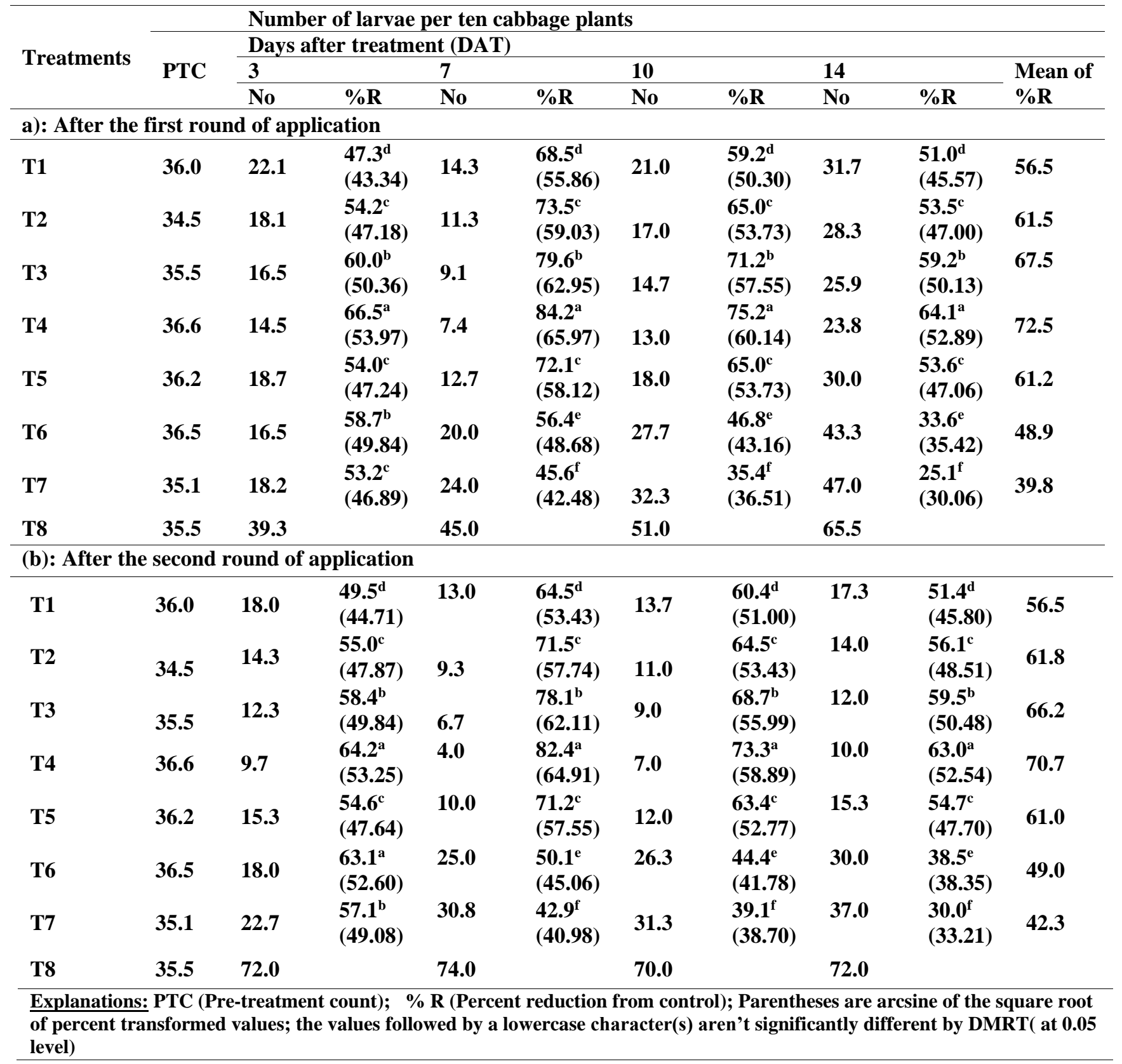


Table 2. The larval population of $P$. xylostella after the third and fourth rounds of application in the first field experiment of the 2019 season.

\begin{tabular}{|c|c|c|c|c|c|c|c|c|c|c|}
\hline \multirow{4}{*}{ Treatments } & \multicolumn{9}{|c|}{ Number of larvae per ten cabbage plants } & \multirow{4}{*}{$\begin{array}{l}\text { Mean } \\
\text { of } \% R\end{array}$} \\
\hline & \multirow{3}{*}{$\begin{array}{l}\text { PTC } \\
(21 \text { DAIIT) }\end{array}$} & \multicolumn{8}{|c|}{ Days after treatment (DAT) } & \\
\hline & & \multicolumn{2}{|l|}{3} & \multicolumn{2}{|c|}{7} & \multicolumn{2}{|l|}{10} & \multicolumn{2}{|l|}{14} & \\
\hline & & No & $\% \mathbf{R}$ & No & $\% \mathbf{R}$ & No & $\% \mathbf{R}$ & No & $\% \mathbf{R}$ & \\
\hline \multicolumn{11}{|c|}{ (a): After the third round of application } \\
\hline T1 & 37.0 & 14.7 & $\begin{array}{l}55.4^{\mathrm{e}} \\
(48.10)\end{array}$ & 11.0 & $\begin{array}{l}68.5^{d} \\
(55.86)\end{array}$ & 15.7 & $\begin{array}{l}59.9^{d} \\
(50.71)\end{array}$ & 25.7 & $\begin{array}{l}44.4^{d} \\
(41.78)\end{array}$ & 57.2 \\
\hline $\mathbf{T 2}$ & 35.0 & 11.0 & $\begin{array}{l}\mathbf{6 4 . 6}^{\mathrm{c}} \\
(53.49)\end{array}$ & 8.5 & $\begin{array}{l}73.5^{\mathrm{c}} \\
(59.22)\end{array}$ & 12.7 & $\begin{array}{l}65.7^{\mathrm{c}} \\
(54.16)\end{array}$ & 22.0 & $\begin{array}{l}49.7^{\mathrm{c}} \\
(\mathbf{4 4 . 8 3 )}\end{array}$ & 63.4 \\
\hline T3 & 33.7 & 9.0 & $\begin{array}{l}69.9^{b} \\
(56.73)\end{array}$ & 6.0 & $\begin{array}{l}\text { 81.1 }^{b} \\
(64.25)\end{array}$ & 11.0 & $\begin{array}{l}69.0^{b} \\
(56.17)\end{array}$ & 19.7 & $\begin{array}{l}53.2^{b} \\
(46.84)\end{array}$ & 68.3 \\
\hline T4 & 36.3 & 7.3 & $\begin{array}{l}77.4^{\mathrm{a}} \\
(61.62)\end{array}$ & 3.0 & $\begin{array}{l}89.2^{\mathrm{a}} \\
(70.03)\end{array}$ & $\mathbf{1 0 . 0}$ & $\begin{array}{l}73.9^{\mathrm{a}} \\
(59.28)\end{array}$ & 18.0 & $\begin{array}{l}60.3^{\mathrm{a}} \\
(50.95)\end{array}$ & 75.2 \\
\hline T5 & 35.3 & 11.3 & $\begin{array}{l}64.0^{c} \\
(53.13)\end{array}$ & 8.5 & $\begin{array}{l}73.5^{c} \\
(58.70)\end{array}$ & 13.0 & $\begin{array}{l}65.1^{c} \\
(53.79)\end{array}$ & 22.7 & $\begin{array}{l}48.6^{c} \\
(44.20)\end{array}$ & 62.8 \\
\hline T6 & 36.0 & 13.0 & $\begin{array}{l}59.4^{d} \\
(50.42)\end{array}$ & 16.3 & $\begin{array}{l}52.0^{\mathrm{e}} \\
(46.15)\end{array}$ & 21.0 & $\begin{array}{l}44.7^{\mathrm{e}} \\
(41.96)\end{array}$ & 29.0 & $\begin{array}{l}35.6^{\mathrm{e}} \\
(\mathbf{3 6 . 6 3 )}\end{array}$ & 48.0 \\
\hline T7 & 34.7 & 14.0 & $\begin{array}{l}54.6 \\
(47.64)\end{array}$ & 18.3 & $\begin{array}{l}44.1^{\mathrm{f}} \\
(41.61)\end{array}$ & 23.3 & $\begin{array}{l}36.3^{f} \\
(37.05)\end{array}$ & 31.0 & $\begin{array}{l}28.5^{f} \\
(32.26)\end{array}$ & 41.0 \\
\hline T8 & 36.0 & 32.0 & & 34.0 & & 38.0 & & 45.0 & & \\
\hline \multicolumn{11}{|c|}{ (b): After the fourth round of application } \\
\hline $\mathrm{T} 1$ & 37.0 & 13.0 & $\begin{array}{l}53.3^{d} \\
(46.89)\end{array}$ & 9.0 & $\begin{array}{l}71.3^{d} \\
(57.61)\end{array}$ & 12.6 & $\begin{array}{l}61.9^{d} \\
(52.18)\end{array}$ & 16.0 & $\begin{array}{l}54.1^{d} \\
(47.35)\end{array}$ & 61.2 \\
\hline $\mathbf{T} 2$ & 35.0 & 9.7 & $\begin{array}{l}59.3^{c} \\
(50.36)\end{array}$ & 5.3 & $\begin{array}{l}80.3^{c} \\
(63.67)\end{array}$ & 8.9 & $\begin{array}{l}67.5^{c} \\
(55.50)\end{array}$ & 12.0 & $\begin{array}{l}59.8^{c} \\
(50.66)\end{array}$ & 66.7 \\
\hline T3 & 33.7 & 8.3 & $\begin{array}{l}61.1 b^{c} \\
(51.42)\end{array}$ & 3.5 & $\begin{array}{l}84.1^{b} \\
(65.98)\end{array}$ & 7.1 & $\begin{array}{l}71.0^{b} \\
(57.36)\end{array}$ & 9.5 & $\begin{array}{l}63.4^{b} \\
(52.95)\end{array}$ & 70.0 \\
\hline T4 & 36.3 & 5.0 & $\begin{array}{l}74.3^{\mathrm{a}} \\
(59.55)\end{array}$ & 1.5 & $\begin{array}{l}91.0^{\mathrm{a}} \\
(72.48)\end{array}$ & 4.9 & $\begin{array}{l}78.5^{a} \\
(62.18)\end{array}$ & 7.5 & $\begin{array}{l}67.9^{a} \\
(55.06)\end{array}$ & 78.0 \\
\hline T5 & 35.3 & 10.0 & $\begin{array}{l}59.3^{\mathrm{c}} \\
(\mathbf{5 0 . 3 6})\end{array}$ & 5.3 & $\begin{array}{l}80.9^{\mathrm{c}} \\
(64.10)\end{array}$ & 9.9 & $\begin{array}{l}66.5^{\mathrm{c}} \\
(54.58)\end{array}$ & 12.3 & $\begin{array}{l}\text { 60.0 } \\
(50.77)\end{array}$ & 66.7 \\
\hline T6 & 36.0 & 12.0 & $\begin{array}{l}\mathbf{6 1 . 8}^{\mathrm{b}} \\
(51.83)\end{array}$ & 16.7 & $\begin{array}{l}52.9^{\mathrm{e}} \\
(46.66)\end{array}$ & 20.8 & $\begin{array}{l}43.4^{\mathrm{e}} \\
(41.03)\end{array}$ & 26.0 & $\begin{array}{l}34.9^{\mathrm{e}} \\
(36.63)\end{array}$ & 55.8 \\
\hline T7 & 34.7 & 15.0 & $\begin{array}{l}55.3^{d} \\
(48.04)\end{array}$ & 18.7 & $\begin{array}{l}5^{50.6}{ }^{\mathrm{e}} \\
(45.34)\end{array}$ & 24.0 & $\begin{array}{l}36.5^{\mathrm{f}} \\
(37.28)\end{array}$ & 33.1 & $\begin{array}{l}24.0^{f} \\
(29.19)\end{array}$ & 49.9 \\
\hline T8 & 36.0 & 48.7 & & 55.0 & & 57.3 & & 61.0 & & \\
\hline
\end{tabular}

Explanations: PTC (Pre-treatment count); DAIIT (Days after second treatment); \% R- Percent reduction from control; Lowercase letters aren't significantly at $\mathbf{P}=0.05$. 
Table 3. The number of diamondback moth's larvae after the first and second rounds of application in the second field experiment (Season of 2020).

\begin{tabular}{|c|c|c|c|c|c|c|c|c|c|c|}
\hline \multirow{4}{*}{ Treatments } & \multicolumn{10}{|c|}{ Number of larvae per ten cabbage plants } \\
\hline & \multirow{3}{*}{ PTC } & \multicolumn{9}{|c|}{ Days after treatment (DAT) } \\
\hline & & \multicolumn{2}{|l|}{3} & \multicolumn{2}{|l|}{7} & \multicolumn{2}{|l|}{10} & \multicolumn{2}{|l|}{14} & \multirow{2}{*}{$\begin{array}{l}\text { Mean of } \\
\% \mathrm{R}\end{array}$} \\
\hline & & No & $\% \mathbf{R}$ & No & $\% \mathbf{R}$ & No & $\% \mathbf{R}$ & No & $\% \mathbf{R}$ & \\
\hline \multicolumn{11}{|c|}{ (a): After the first round of application } \\
\hline $\mathbf{T 1}$ & 57.1 & 31.0 & $\begin{array}{l}52.9^{d} \\
(46.66)\end{array}$ & 22.3 & $\begin{array}{l}71.4^{d} \\
(57.68)\end{array}$ & 34.7 & $\begin{array}{l}60.4^{d} \\
(51.00)\end{array}$ & 50.0 & $\begin{array}{l}49.9^{d} \\
(44.94)\end{array}$ & 58.65 \\
\hline $\mathbf{T} 2$ & 54.0 & 27.3 & $\begin{array}{l}56.7^{c} \\
(48.85)\end{array}$ & 18.7 & $\begin{array}{l}75.0^{c} \\
(60.01)\end{array}$ & 28.7 & $\begin{array}{l}65.8^{c} \\
(54.22)\end{array}$ & 45.7 & $\begin{array}{l}52.3^{c} \\
(46.32)\end{array}$ & 62.45 \\
\hline $\mathbf{T 3}$ & 52.5 & 24.0 & $\begin{array}{l}63.1^{b} \\
(52.54)\end{array}$ & 13.7 & $\begin{array}{l}82.1^{b} \\
(64.47)\end{array}$ & 24.0 & $\begin{array}{l}70.9^{b} \\
(57.36)\end{array}$ & 40.0 & $\begin{array}{l}57.6^{b} \\
(49.26)\end{array}$ & 68.42 \\
\hline $\mathbf{T 4}$ & 54.7 & 21.0 & $\begin{array}{l}66.9^{a} \\
(55.06)\end{array}$ & 12.0 & $\begin{array}{l}83.9^{a} \\
(66.59)\end{array}$ & 22.0 & $\begin{array}{l}74.1^{\mathrm{a}} \\
(59.41)\end{array}$ & 37.0 & $\begin{array}{l}62.1^{\mathrm{a}} \\
(51.83)\end{array}$ & 71.75 \\
\hline T5 & 55.7 & 28.3 & $\begin{array}{l}56.5^{c} \\
(48.74)\end{array}$ & 20.0 & $\begin{array}{l}74.1^{\mathrm{c}} \\
(59.41)\end{array}$ & 30.0 & $\begin{array}{l}65.3^{c} \\
(53.91)\end{array}$ & 48.3 & $\begin{array}{l}51.0^{\text {cd }} \\
(45.57)\end{array}$ & 61.73 \\
\hline T6 & 54.7 & 23.7 & $\begin{array}{l}61.5^{b} \\
(51.53)\end{array}$ & 31.0 & $\begin{array}{l}59.1^{\mathrm{e}} \\
(\mathbf{5 0 . 2 5 )}\end{array}$ & 43.3 & $\begin{array}{l}49.0^{\mathrm{e}} \\
(44.43)\end{array}$ & 64.3 & $\begin{array}{l}33.6^{\mathrm{e}} \\
(35.42)\end{array}$ & 50.80 \\
\hline T7 & 53.7 & 26.7 & $\begin{array}{l}57.5^{\mathrm{c}} \\
(49.31)\end{array}$ & 38.0 & $\begin{array}{l}48.9^{f} \\
(44.37)\end{array}$ & 55.0 & $\begin{array}{l}34.1^{\mathrm{f}} \\
(35.73)\end{array}$ & 72.0 & $\begin{array}{l}24.3^{f} \\
(29.53)\end{array}$ & 41.2 \\
\hline T8 & 55.3 & 64.7 & & 76.7 & & 86.0 & & 98.0 & & \\
\hline \multicolumn{11}{|c|}{ (b): After the second round of application } \\
\hline T1 & 57.1 & 22.0 & $\begin{array}{l}58.5^{\mathrm{e}} \\
(49.90)\end{array}$ & 18.0 & $\begin{array}{l}\mathbf{6 8 . 5}^{\mathrm{d}} \\
(55.86)\end{array}$ & 22.0 & $\begin{array}{l}58.0^{d} \\
(49.61)\end{array}$ & 24.0 & $\begin{array}{l}45.5^{d} \\
(42.42)\end{array}$ & 57.63 \\
\hline $\mathbf{T} 2$ & 54.0 & 18.0 & $\begin{array}{l}62.9^{c} \\
(52.48)\end{array}$ & 14.0 & $\begin{array}{l}73.2^{c} \\
(58.83)\end{array}$ & $\mathbf{1 7 . 3}$ & $\begin{array}{l}63.8^{c} \\
(53.02)\end{array}$ & 19.0 & $\begin{array}{l}52.8^{c} \\
(46.61)\end{array}$ & 63.18 \\
\hline T3 & 52.5 & 14.7 & $\begin{array}{l}65.4^{b} \\
(53.97)\end{array}$ & 9.0 & $\begin{array}{l}80.3^{b} \\
(63.66)\end{array}$ & 13.7 & $\begin{array}{l}67.6^{b} \\
(55.13)\end{array}$ & 15.3 & $\begin{array}{l}56.5^{b} \\
(48.74)\end{array}$ & 67.45 \\
\hline T4 & 54.7 & 12.0 & $\begin{array}{l}69.4^{\mathrm{a}} \\
(56.42)\end{array}$ & 6.7 & $\begin{array}{l}84.5^{a} \\
(66.59)\end{array}$ & 11.3 & $\begin{array}{l}71.3^{a} \\
(57.30)\end{array}$ & 13.0 & $\begin{array}{l}60.1^{\mathrm{a}} \\
(50.83)\end{array}$ & 71.33 \\
\hline T5 & 55.7 & 19.7 & $\begin{array}{l}61.6^{\text {cd }} \\
(51.71)\end{array}$ & 15.3 & $\begin{array}{l}72.3^{c} \\
(58.25)\end{array}$ & 18.7 & $\begin{array}{l}63.0^{c} \\
(52.54)\end{array}$ & 20.7 & $\begin{array}{l}51.3^{c} \\
(45.75)\end{array}$ & 62.05 \\
\hline T6 & 54.7 & 27.0 & $\begin{array}{l}60.5^{\mathrm{d}} \\
(51.06)\end{array}$ & 32.0 & $\begin{array}{l}56.5^{\mathrm{e}} \\
(48.74)\end{array}$ & 35.3 & $\begin{array}{l}47.6^{\mathrm{e}} \\
(43.62)\end{array}$ & 38.0 . & $\begin{array}{l}32.9^{\mathrm{e}} \\
(35.00)\end{array}$ & 49.38 \\
\hline T7 & 53.7 & 33.0 & $\begin{array}{l}56.8^{\mathrm{e}} \\
(48.91)\end{array}$ & 44.7 & $\begin{array}{l}44.8^{f} \\
(42.53)\end{array}$ & 46.7 & $\begin{array}{l}38.1^{\text {f }} \\
(38.11)\end{array}$ & 49.3 & $\begin{array}{l}22.3 \text { f } \\
(28.17)\end{array}$ & 40.50 \\
\hline T8 & 55.3 & 104.0 & & 112.0 & & 102.7 & & 86.3 & & \\
\hline
\end{tabular}

Explanations: PTC (Pre-treatment count); DAIIT (Days after second treatment); \% R- Percent reduction from control; Lowercase letters aren't significantly at $\mathrm{P}=0.05$. 
Table 4. Number of $P$. xylostella's larvae after the third and fourth round of application in the second field experiment of 2020 season.

\begin{tabular}{|c|c|c|c|c|c|c|c|c|c|c|}
\hline \multirow{4}{*}{ Treatments } & \multicolumn{10}{|c|}{ Number of larvae per ten cabbage plants } \\
\hline & \multirow{3}{*}{$\begin{array}{l}\text { PTC } \\
\text { (21DAIIT) }\end{array}$} & \multicolumn{9}{|c|}{ Days after treatment (DAT) } \\
\hline & & \multirow{2}{*}{\begin{tabular}{|l|}
3 \\
No \\
\end{tabular}} & \multicolumn{2}{|c|}{7} & \multicolumn{2}{|c|}{10} & \multicolumn{2}{|l|}{14} & \multirow[b]{2}{*}{$\% \mathbf{R}$} & \multirow{2}{*}{$\begin{array}{l}\text { Mean of } \\
\% R\end{array}$} \\
\hline & & & $\% \mathbf{R}$ & No & $\% \mathbf{R}$ & No & $\% \mathbf{R}$ & No & & \\
\hline \multicolumn{11}{|c|}{ (a): After the third round of application } \\
\hline $\mathbf{T 1}$ & 55.3 & $\begin{array}{l}23 . \\
0\end{array}$ & $\begin{array}{l}59.9^{\mathrm{e}} \\
(50.71)\end{array}$ & 14.0 & $\begin{array}{l}76.4^{d} \\
(60.94)\end{array}$ & 21.0 & $\begin{array}{l}65.7^{d} \\
(54.15)\end{array}$ & 28.0 & $\begin{array}{l}55.2^{d} \\
(47.99)\end{array}$ & 64.3 \\
\hline $\mathbf{T} 2$ & 53.7 & 18. & $\begin{array}{l}67.6^{c} \\
(55.31)\end{array}$ & 11.0 & $\begin{array}{l}81.2^{c} \\
(64.11)\end{array}$ & 17.3 & $\begin{array}{l}70.9^{c} \\
(57.36)\end{array}$ & 23.7 & $\begin{array}{l}60.9^{c} \\
(51.30)\end{array}$ & 70.08 \\
\hline $\mathbf{T 3}$ & 52.7 & 13. & $\begin{array}{l}74.9^{b} \\
(59.94)\end{array}$ & 7.0 & $\begin{array}{l}87.6^{b} \\
(69.41)\end{array}$ & 15.0 & $\begin{array}{l}74.3^{b} \\
(59.55)\end{array}$ & 21.0 & $\begin{array}{l}64.7^{b} \\
(53.55)\end{array}$ & 75.38 \\
\hline T4 & 54.0 & 11. & $\begin{array}{l}80.3^{a} \\
(63.66)\end{array}$ & 5.0 & $\begin{array}{l}92.5^{a} \\
(72.99)\end{array}$ & 12.0 & $\begin{array}{l}79.9^{a} \\
(63.37)\end{array}$ & 20.0 & $\begin{array}{l}67.2^{\mathrm{a}} \\
(55.06)\end{array}$ & 79.96 \\
\hline T5 & $\mathbf{5 9 . 0}$ & 19. & $\begin{array}{l}67.8^{c} \\
(55.43)\end{array}$ & 12.3 & $\begin{array}{l}80.9^{c} \\
(63.80)\end{array}$ & 20.0 & $\begin{array}{l}69.4^{c} \\
(56.42)\end{array}$ & 26.0 & $\begin{array}{l}61.0^{c} \\
(51.36)\end{array}$ & 69.78 \\
\hline T6 & 54.0 & $\begin{array}{l}21 . \\
0\end{array}$ & $\begin{array}{l}62.5^{\mathrm{d}} \\
(52.24)\end{array}$ & 26.0 & $\begin{array}{l}55.1^{\mathrm{e}} \\
(\mathbf{4 7 . 9 3 )}\end{array}$ & 34.0 & $\begin{array}{l}43.1^{\mathrm{e}} \\
(41.03)\end{array}$ & 38.0 & $\begin{array}{l}37.7^{\mathrm{e}} \\
(\mathbf{3 7 . 8 8})\end{array}$ & 49.6 \\
\hline T7 & 55.3 & 24. & $\begin{array}{l}58.1^{\mathrm{e}} \\
(49.66)\end{array}$ & 30.0 & $\begin{array}{l}49.4^{\mathrm{f}} \\
(44.66)\end{array}$ & 38.7 & $\begin{array}{l}36.8^{\mathrm{f}} \\
(37.34)\end{array}$ & 42.0 & $\begin{array}{l}32.8^{\mathrm{f}} \\
(34.94)\end{array}$ & 44.28 \\
\hline T8 & 56.0 & $\begin{array}{l}58 . \\
0 \\
\end{array}$ & & 60.0 & & 62.7 & & 63.3 & & \\
\hline \multicolumn{11}{|c|}{ (b): After the fourth round of application } \\
\hline $\mathbf{T 1}$ & 55.3 & $\begin{array}{l}10 . \\
3\end{array}$ & $\begin{array}{l}63.6^{d} \\
(52.89)\end{array}$ & 8.7 & $\begin{array}{l}6^{69.6^{d}} \\
(56.54)\end{array}$ & 11.0 & $\begin{array}{l}6^{62.1^{d}} \\
(51.77)\end{array}$ & 12.3 & $\begin{array}{l}57.2^{d} \\
(49.14)\end{array}$ & 63.13 \\
\hline $\mathbf{T} 2$ & 53.7 & 8.0 & $\begin{array}{l}66.6^{c} \\
(54.70)\end{array}$ & 5.3 & $\begin{array}{l}78.1^{\mathrm{c}} \\
(62.11)\end{array}$ & 7.3 & $\begin{array}{l}70.2^{\mathrm{c}} \\
(56.80)\end{array}$ & 9.0 & $\begin{array}{l}63.0^{\mathrm{c}} \\
(52.54)\end{array}$ & 69.48 \\
\hline T3 & 52.7 & 6.3 & $\begin{array}{l}70.3^{b} \\
(56.98)\end{array}$ & 3.3 & $\begin{array}{l}84.6^{b} \\
(66.92)\end{array}$ & 5.0 & $\begin{array}{l}77.1^{b} \\
(61.22)\end{array}$ & 7.3 & $\begin{array}{l}66.5^{b} \\
(54.40)\end{array}$ & 74.63 \\
\hline T4 & 54.0 & 5.0 & $\begin{array}{l}75.3^{a} \\
(60.21)\end{array}$ & 2.0 & $\begin{array}{l}90.2^{a} \\
(71.79)\end{array}$ & 3.0 & $\begin{array}{l}86.0^{a} \\
(67.56)\end{array}$ & 6.0 & $\begin{array}{l}71.0^{\mathrm{a}} \\
(57.30)\end{array}$ & 80.63 \\
\hline T5 & 59.0 & 9.0 & $\begin{array}{l}65.8^{c} \\
(54.21)\end{array}$ & 5.7 & $\begin{array}{l}78.6^{c} \\
(62.45)\end{array}$ & 7.7 & $\begin{array}{l}71.2^{c} \\
(57.55)\end{array}$ & 10.0 & $\begin{array}{l}62.5^{\mathrm{c}} \\
(52.24)\end{array}$ & 69.53 \\
\hline T6 & 54.0 & $\begin{array}{l}14 . \\
3\end{array}$ & $\begin{array}{l}62.8^{d} \\
(52.42)\end{array}$ & 19.0 & $\begin{array}{l}51.1^{\mathrm{e}} \\
(45.63)\end{array}$ & 20.0 & $\begin{array}{l}49.0^{\mathrm{e}} \\
(44.25)\end{array}$ & 24.0 & $\begin{array}{l}38.7^{\mathrm{e}} \\
(\mathbf{3 8 . 3 5})\end{array}$ & 50.40 \\
\hline T7 & 55.3 & 19. & $\begin{array}{l}54.6^{\mathrm{e}} \\
(47.64)\end{array}$ & 23.0 & $\begin{array}{l}46.4^{\mathrm{f}} \\
(42.94)\end{array}$ & 24.7 & $\begin{array}{l}43.0^{f} \\
(40.80)\end{array}$ & 28.0 & $\begin{array}{l}34.9^{f} \\
(36.33)\end{array}$ & 45.18 \\
\hline T8 & 56.0 & $\begin{array}{l}64 . \\
0\end{array}$ & & 64.7 & & 65.0 & & 65.0 & & \\
\hline
\end{tabular}

Explanations: PTC (Pre-treatment count); DAIIT (Days after second treatment); \% R- Percent reduction from control; Lowercase letters aren't significantly at $\mathbf{P}=0.05$.

The present findings are by following the reports of Rui (2001) who found that the effect of abamectin was lesser one day after application, but rapidly increased three days after application. However, on 7 DAT, abamectin $(1.5 \%)+$ Bt WP at a dilution rate of $1: 750$ and 1500 and abamectin $(0.9 \%$ EC) at a dilution rate of 1:3000 showed 90.9 percent control of DBM. These results were also found by Yan et al. (2001), Elzen and James (2002), Pramanik and Chatterjee (2003), and Sawant and Patil (2017) who have reported the effectiveness of abamectin against the diamondback moth.
The treated cabbage plants with spinosad were in line with abamectin at $11 \mathrm{~g}$ a.i./ ha throughout the study period in the experiments. Spinosad registered $72.1,71.2,73.0$, and $80.9 \%$ reduction in the population of DBM across the first, second, third, and fourth spray application in the first experiment on 7 DAT, respectively. The same trend of efficacy was seen in the second experiment also (Figs. 2 and 3). The obtained data approves the findings of Walunj et al. (2001) who found that spinosad $2.5 \% \mathrm{SC}$ at the doses of 12.5 , 15 , and $17.5 \mathrm{~g}$ a.i./ ha on cabbage resulted in the lowest larval population of 0.47 to 2.27 larvae plant $^{-1}$ as against 5.3 to 6.73 larvae plant $^{-1}$ in the control. The efficacy of spinosad against DBM has 
been stated by Yan et al. (2001), and Syed et al. (2004). Vaseemet al., 2014, Stanikzi and Thakur, 2016, Reddy et al., 2017, and Sharma et al., 2017.

The treated plant with Cypermethrin registered a maximum reduction in larval population (58.4, $63.1,59.4$, and $61.8 \%$ ) while the plants treated with endosulfan recorded 3.3, 57.1, 54.6, and 55.3 percent from untreated check after the first, second, third, and fourth spray, respectively in the first experiment on 3 DAT. Thereafter, the efficacy was drastically reduced in these treatments. The same trend of efficacy was observed in the second experiment too. The present findings agree with the reports of Walunj et al. (2001), Umashankar and Raju (2002), Sakthi et al. (2003), Bhavani and Punnaiah (2004), Chandrasekhar and Marutiram (2004), and Ojha et al. (2004) who reported moderate control of DBM was obtained with cypermethrin and endosulfan. Legwaila et al. (2014) showed that cypermethrin can still be used to achieve effective control of DBM eggs and larvae.

\section{Effects on Yield}

\section{a) First experiment (Season of 2019).}

The highest yield (37.6 tonnes/ha) of marketable cabbage heads with an increase of $60 \%$ was obtained with abamectin at $15 \mathrm{~g}$ a.i./ha over the untreated check. Abamectin at $11 \mathrm{~g}$ a.i./ ha recorded 34.3 tonnes/ha, which was on par with spinosad at $75 \mathrm{~g}$ a.i./ ha (34.0 tonnes/ha), whereas the control treatment registered the lowest yield of 23.5 tonnes/ ha (Table 5; Fig. 4). b) Second experiment (Season of 2020).

The highest yield of 37.7 tonnes/ ha of marketable cabbage was realized in abamectin $15 \mathrm{~g}$ a.i/.ha followed by abamectin at $13 \mathrm{~g}$ a.i./ha (36.6 tonnes/ ha). abamectin at $11 \mathrm{~g}$ a.i./ha registered 35.4 tonnes/ha, which was agreed with the obtained yield (35 tonnes/ ha) by $75 \mathrm{~g}$ a.i./ha of spinosad, while cypermethrin $10 \mathrm{EC}$ at $70 \mathrm{~g}$ a.i./ha and endosulfan $35 \mathrm{EC}$ at $420 \mathrm{~g}$ a.i./ha registered 31.6 and 30.8 tonnes $/$ ha, respectively (Fig.4). The control check verified the lowest yield of 25.0 tonnes/ ha (Table 5).

An increase in the yields of marketable cabbage heads was recorded in all the abamectin treatments which ranged from 33.2 to 37.6 tonnes/ ha and 33.6 to 37.7 tonnes /ha in the first and second experiments, respectively while the untreated check recorded 23.5 and 25.0 tonnes /ha, respectively (Fig. 4). It is therefore concluded that abamectin at 11,13 , and $15, \mathrm{~g}$ a.i./ha was more operative and effective against $P$. xylostella and these results conform with the earlier results of Murugan and Ramachandran (2000) who found that Vertimec®1.8 EC @ 15 and $20 \mathrm{~g}$ a.i./ha recorded 51 and 52 tonnes /ha of marketable cabbage heads, respectively while the control treatment registered 33 tonnes per ha of yield. Spinosad $45 \mathrm{SC}$ at $75 \mathrm{~g}$ a.i./ ha recorded 34 and 35 tonnes/ ha in the first and second field experiments, respectively (Fig.4). This was in agreement with the findings of Walunj et al. (2001) that spinosad 2.5 SC at $15 \mathrm{~g}$ a.i.ha-1 recorded 58.12 tonnes/ ha.

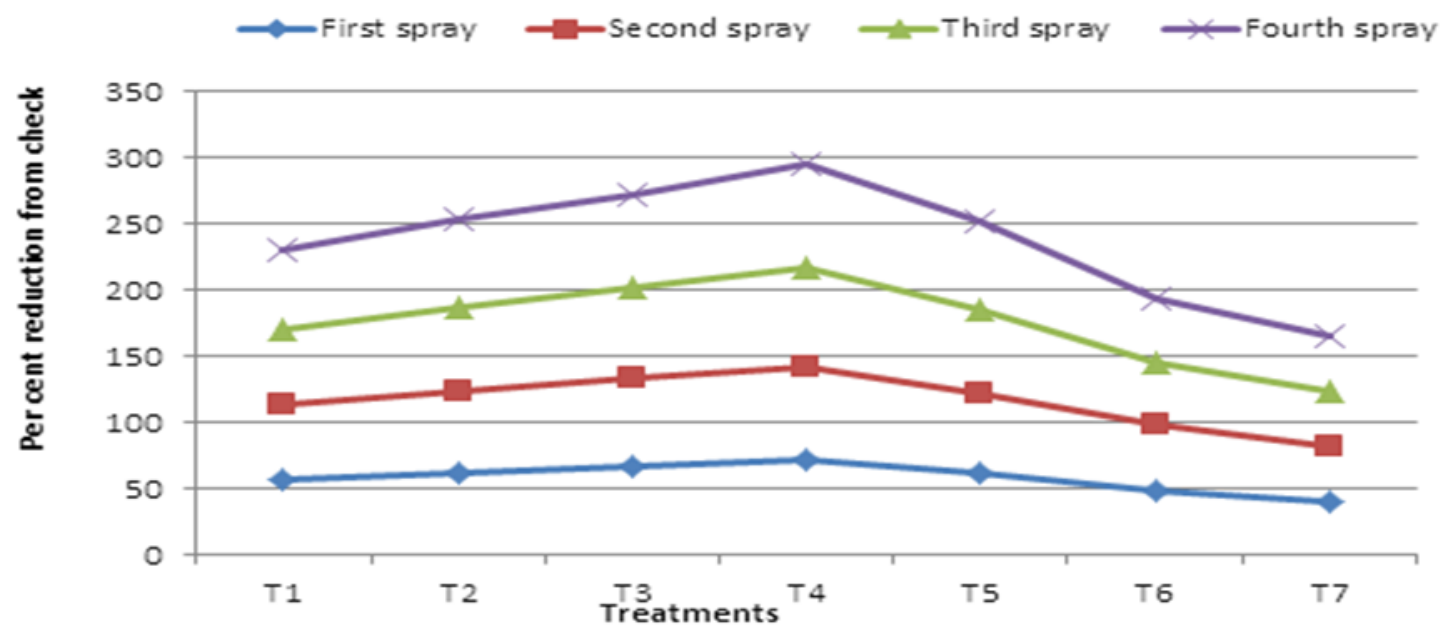

Fig. 2. Effect of abamectin 1.8 EC on the larval population of Plutella xylostella L. on cabbage in the first experiment (Season of 2019). 


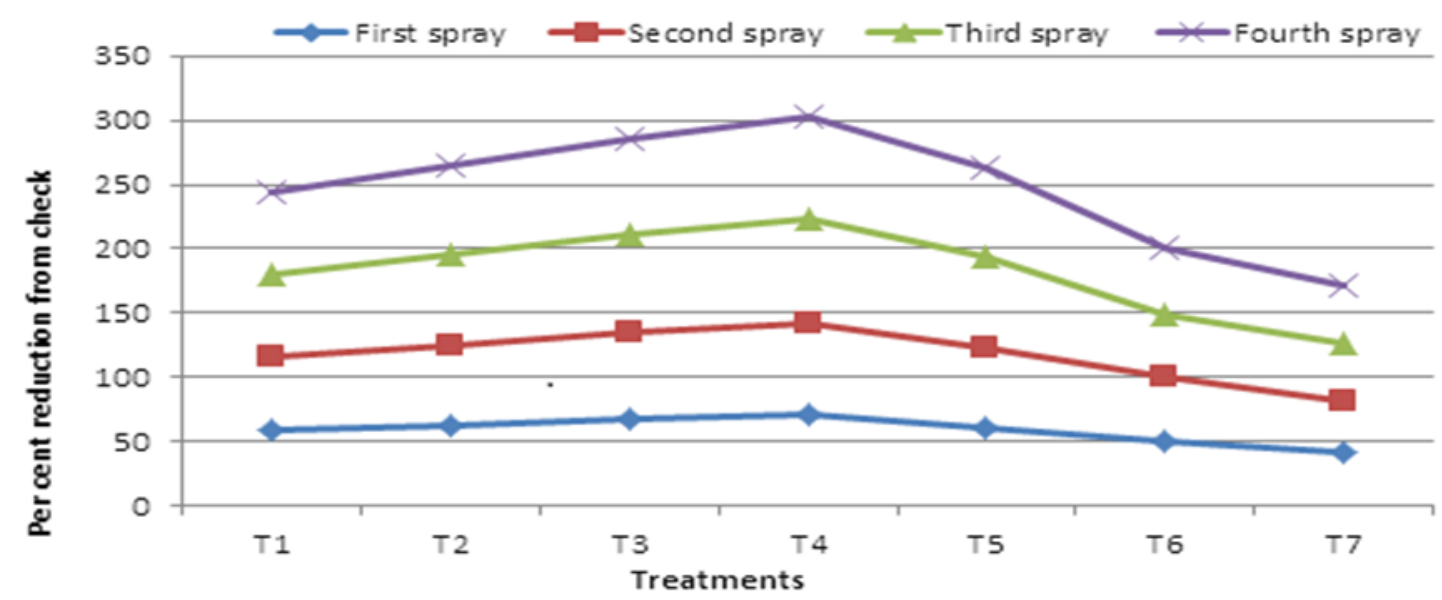

Fig. 3. Effect of abamectin 1.8 EC on the larval population of P. xylostellaon cabbage based on the second experiment (Season of 2020).

Table 5. Effect of abamectin 1.8 EC on cabbage yield in the two field experiments.

\begin{tabular}{|c|c|c|c|c|c|c|}
\hline \multirow{2}{*}{ Treatments } & \multicolumn{3}{|c|}{ Yield in the first experiment } & \multicolumn{3}{|c|}{ Yield in the second experiment } \\
\hline & Kg plot ${ }^{-1}$ & Tonnes ha $^{-1}$ & $\begin{array}{l}\text { Percent increase } \\
\text { over control }\end{array}$ & Kg plot ${ }^{-1}$ & Tonnes ha $^{-1}$ & $\begin{array}{l}\text { Percent increase } \\
\text { over control }\end{array}$ \\
\hline T1 & $66.4^{\text {cd }}$ & 33.2 & 35.1 & $67.2^{\text {cd }}$ & 33.6 & 34.4 \\
\hline $\mathbf{T} 2$ & $68.6^{\mathrm{bc}}$ & 34.3 & 45.9 & $70.8^{\text {abc }}$ & 35.4 & 41.6 \\
\hline T3 & $71.0^{\mathrm{ab}}$ & 35.5 & 51.1 & $73.2^{\mathrm{ab}}$ & 36.6 & 46.4 \\
\hline T4 & $75.2^{\mathrm{a}}$ & 37.6 & 60.0 & $75.4^{\mathrm{a}}$ & 37.7 & 50.8 \\
\hline $\mathbf{T 5}$ & $68.0^{\mathrm{bc}}$ & 34.0 & 44.7 & $70.0^{\mathrm{bc}}$ & 35.0 & 40.0 \\
\hline T6 & $63.6^{\mathrm{cd}}$ & 31.8 & 35.3 & $63.2^{\text {de }}$ & 31.6 & 26.4 \\
\hline T7 & $62.0^{\mathrm{d}}$ & 31.0 & 31.9 & $61.6^{\mathrm{ef}}$ & 30.8 & 23.2 \\
\hline T8 & $47.0^{\mathrm{e}}$ & 23.5 & - & $50.0^{\mathrm{f}}$ & 25.0 & - \\
\hline
\end{tabular}

Explanation: Lowercase letter(s) followed the mean values aren't significantly different by DMRT at $P=0.05$ level.

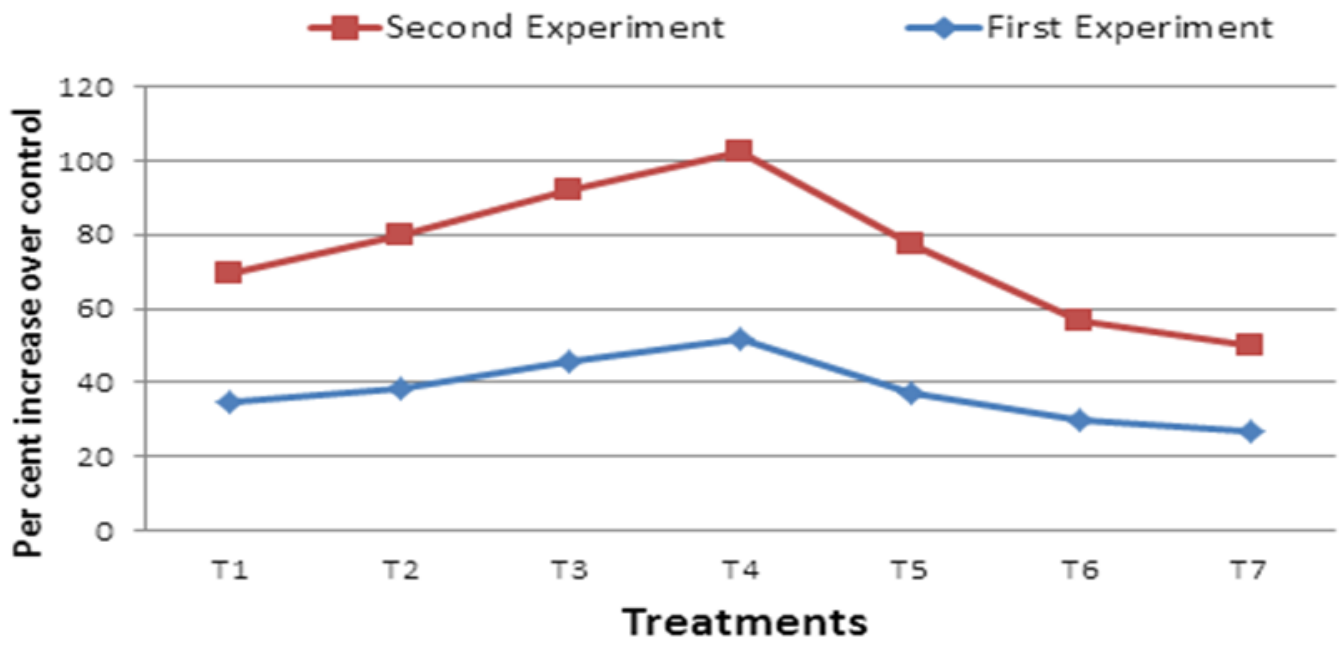

Fig. 4. The increase over control of abamectin 1.8 EC treatments in cabbage yield in the two experiments (Seasons of 2019 and 2020). 


\section{CONCLUSION}

Two field experiments in different seasons were conducted at El-Kattawia area, Sharkia Governorate, Egypt to evaluate the bioefficacy of abamectin $1.8 \mathrm{EC}$ against cabbage diamondback moth (Plutella xylostella $\mathrm{L}$ ). The results of the experiment revealed that abamectin at $15 \mathrm{~g}$ a.i./ ha registered the highest mean reduction of 772.5 , $70.7,75.2$, and 78.0 percent of diamondback moth population from untreated check after first, second, third, and fourth sprays, respectively. But, abamectin at $11 \mathrm{~g}$ a.i./ ha also significantly suppressed the population of diamondback moth larvae and recorded a mean reduction of 61.5, 61.8, 63.4, and 66.7 percent from untreated check after first, second, third, and four rounds of sprays, respectively and was on par with standard check spinosad $75 \mathrm{~g}$ a.i./ha and excelled over cypermethrin and endosulfan. A similar trend was also observed in the second experiment. Abamectin resulted in increased cabbage yield at all the doses tested in the experiments. The effective order of relative efficacy was $15>13>11$ $\mathrm{g}$ a.i./ha of abamectin1.8 $\mathrm{EC} \geq 75 \mathrm{~g}$ a.i./ha of spinosad $45 \mathrm{SC}>9 \mathrm{~g}$ a.i./ha of abamectin $>70 \mathrm{~g}$ a.i./ha of cypermethrin $10 \mathrm{EC}>420 \mathrm{~g}$ a.i./ha of endosulfan $35 \mathrm{EC}$. The field experiments presented a highly positive eco-friendly method of abamectin $1.8 \mathrm{EC}$ at suggested doses to manage the diamondback moth under a semi-arid zone in Egypt.

\section{REFERENCES}

Bhavani, B. and K.C. Punnaiah. 2004. Comparative efficacy of selected insecticides against major pests of cabbage. Pestology, 28(8): 20-27.

Capinera, J. L. 2018. Diamondback moth. Featured Creatures, University of Florida Publication EENY-119.

Chandrasekhar, N. and G. Marutiram. 2004. Bioefficacy of oxymatrine, a plant-derived pesticide, Vs conventional pesticides in the management of DBM Plutella xylostella (L.) on cabbage.Pestology, 28(5):4-7.

Dara, S. K. 2020. Arthropod resistance to biopesticides. Organic Farmer 3 (4): 16-19.

Diab, H. S. T. 2011. Resistance categories of Kalubia and Menufia diamondback moth strains to some insecticides. J. Plant Prot. and Path., Mansoura Univ., Vol. 2 (10): 835 - 843.

Egyptian Meteorological Authority. 2020. Climatic Atlas of Egypt - Meteorological stations, Cairo, Egypt.

Elzen, G.W. and R.R. James. 2002. Response of Plutella xylostella and Coleomegilla maculata to selected insecticides in a residual insecticide bioassay. Southwestern Entomologist, 27(2): 149153.

FAO. 1970. Physical and Chemical Methods of Soil and Water Analysis. Soils Bulletin No.10. FAO, Rome, Italy.

Fauziah, I., M. T. Norazam, M. T. and Z. MohdRasdi. 2012. "Toxicity of Selected Insecticides (Spinosad, Indoxacarb and Abamectin) Against the Diamondback Moth (Plutella xylostella L.) On Cabbage" Asian Journal of Agriculture and Rural Development Economic and Financial Review Vol. 2, No.1, pp.17-26.

Gomez, K.A. and A.A. Gomez. 1976. Statistical Procedures for Agricultural Research with Special Emphasis on rice. International Rice Research Institute, Los Banos, Phillippines.368 p.

Harika G., S. Dhurua, M. Suresh and N. Sreesandhya. 2019. Evaluation of Certain Insecticides against Diamondback Moth (DBM) Plutella xylostella on Cauliflower. International Journal of Bio-resource and Stress Management. 10(1):070-076.

Jackson, M.L. 1973. Soil Chemical Analysis. Advanced course. 2nd ed. A Manual of Methods Useful for Instruction and Research in Soil Chemistry, Physical Chemistry of Soil, Soil Fertility, and Soil Genesis. Revised from Original Edition (1955). U.S.A.

Krishnakumar, N.K., K. Srinivasan, P.R. Ramachadra and C.L. Suman. 1986. Optimum control strategy of cabbage pests from a chemical control trial. Progressive Hort.,18: 104-110.

Legwaila M. M., D. C. Munthali, B. C. Kwerepe and M. Obopile Motshwari (2014). Effectiveness of cypermethrin against diamondback moth (Plutella xylostella L.) eggs and larvae on cabbage under Botswana conditions. Afr. J. Agric. Res. Vol.9(51), pp. 3704-3710

Murugan, M. and K. Ramachandran. 2000. Bioefficacy of Vertimec ${ }^{\circledR} 1.8$ EC against DBM Plutella xylostella (L.) on cabbage. Pestology, 24(1): 12-14.

Ojha, P.K., I. P. Singh and N.K. Pandey. 2004. Comparative efficacy of some new insecticides against insect pests of cauliflower under agroclimatic zone - 1 of Bihar.Pestology, 28(4): 36-39.

Pramanik, P. and M.L. Chatterjee. 2003. Efficacy of some new insecticides in the management of diamond back moth Plutella xylostella L. in cabbage. Indian J. Pl. Prot., 31(2): 42-44.

Reddy, A., Shashi, V., Rao, C.H.S., Aruna, M., 2017. Bio-efficacy of insecticides against diamondback moth Plutella xylostella $\mathrm{L}$. in 
cabbage (Brassica oleraceavar. capitata). International Journal of Current Microbiology and Applied Sciences 6(6), 1121-1125.

Rui, C.W. 2001. Comparative study on efficacy of five biopesticides for the control of Plutellaxylostella.Pl. Prot.,27(6): 33-34.

Sakthi, E., N. Seenivasan, K. Devrajan and N. Selvaraj. 2003. Bioefficacy of new insecticides against cabbage DBM Plutella xylostella L. Pestology, 27(4): 35-37..

Sawant, C.G., Patil, C.S., 2017. Bio-efficacy of newer insecticides against diamondback moth (Plutella xylostella L.) in cabbage. International Journal of Bioengineering and Life Sciences 11(10), 51-54.

Scott, R. and I. Dwe. 1985. Effects of 22, 23 dihydro avermectin B1a on locust Schistocerca gregaria muscles that involve several sites of action. Pestic. Sci., 16: 599-604.

Sengonca, C. and B. Liu. 2001. Influence of mixed biocide GCSC - Bt A on the pupa and adult stages of Apanteles plutellae Kurd (Hymenoptera: Braconidae) and its host, Plutella xylostella (L.) (Lepidoptera: Plutellidae). Bull. Entomol. Res., 7: 51-58.

Sharma, P., Kumawat, K.C., Khinchi, S.K., Kumar, V., Prasad, B., 2017. Bioefficacy of different insecticides against diamondback moth. International Journal of Chemical Studies 5(3), 891-893.

Soil Survey Staff. 2014. Kellogg Soil Survey Laboratory Methods Manual. Soil Survey Investigations Report No. 42, version 5.0. R. Burt and Soil Survey Staff (eds.). USDA Natural Resources Conservation Service.
Stanikzi, R., Thakur, S., 2016. Efficacy of chemical insecticides and botanicals in the management of diamondback moth (Plutella xylostella) in cabbage (Brassica oleraceavar. Capitata L.). International Journal of Multidisciplinary Research and Development 3(6), 101-104.

Syed, T.S., G.H. Abro and S. Ahmed. 2004. Efficacy of different insecticides against Plutella xylostella under field conditions. Pakistan J. Biol. Sci., 7(1): 10-13.

Talekar, N.S. 1990. Development of an integrated pest management program for the control diamondback moth on crucifers vegetables. Vegetable Research and Development in SADCC countries In: Opena, R.T., and Kyomo, M.L. (ed), AVRDC, Shanhua, Taiwan, pp147-157.

Umashankar and S.V.S. Raju. 2002. Bioefficacy of some new insecticides molecules against DBM, Plutella xylostella L. on cauliflower. Pestology, 26(8): 41-46.

Vaseem, M., Singh, H., Kumar, K., Ali, M. 2014. Efficacy of newer insecticides against diamondback moth (Plutella xylostella Linn) on cabbage under polyhouse condition. Journal of Experimental Zoology India 17(2), 487-489.

Walunj, A.R., S.A. Pawar and K.S. Darekar. 2001. Evaluation of a new molecule, spinosad 2.5 SC for the control of DBM (Plutella xylostella L.) on cabbage. Pestology,25(9): 6-7.

Yan, Z.X., H. Jie, Y.C. Yu and X. Yi. 2001. Monitoring on resistance of diamond back moth to abamectin and field control experiments in Yunnan, China. J. Huazhong Agric. Univ., 20(5): 426-430. 
الملخص العربي

\section{استخدام الأبامكتين كأحد المُبيدات الصديقة للبيئة لمُكافحة الفراشة ذات الظهر الماسي على محصول الكرنب}

مريم مُسعد مرسي محمد 1 ، عادل عبدالحميد علوان خليل2

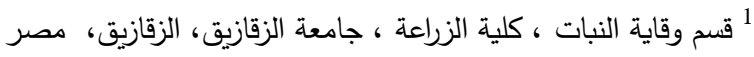
2 قسم البيدولوجي، مركز بحوث الصحراء ، القاهرة ، رقم بريدي 11753 ، 117 ،

تعتبر الفراشة ذات الظهر الماسي Diamondback Moth من أكثر الآفات تدميراً لمحصول الكرنب Cabbage crop في جمهورية

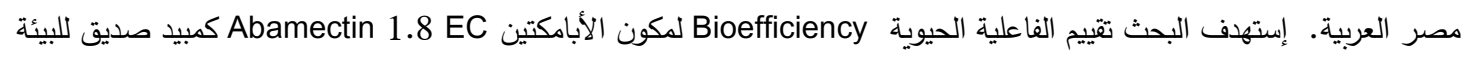
Eco-friendly pesticide

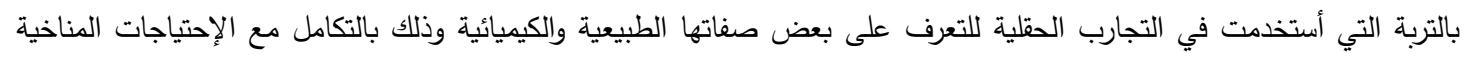
والمائية لمحصول الكرنب تحت الدراسة؛ من أجل التأكيد على توفير البيئة المناسبة والإحتياجات الأساسية لتغذية ونمو نباتات الكرنب التباتية

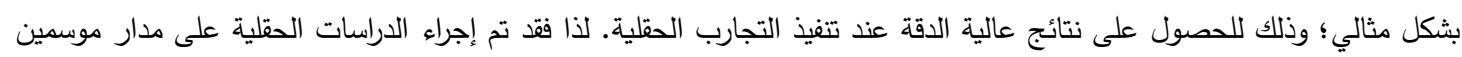

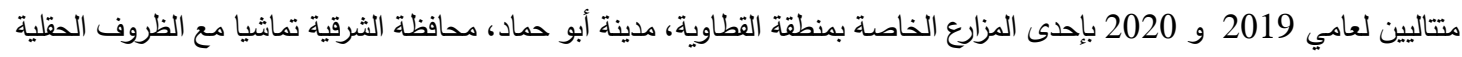

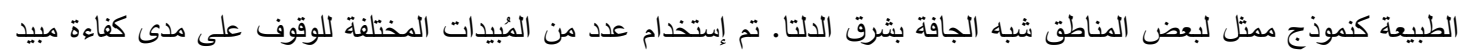

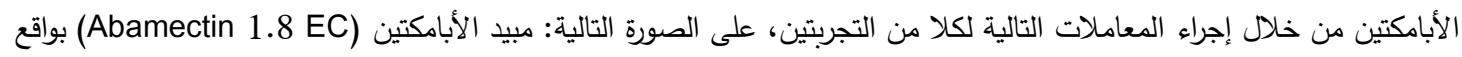

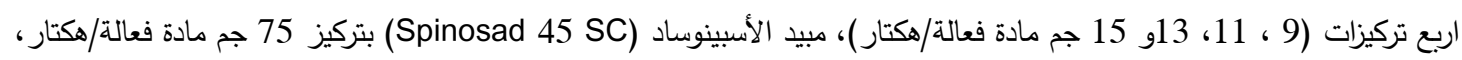

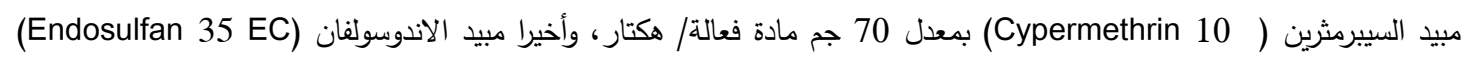

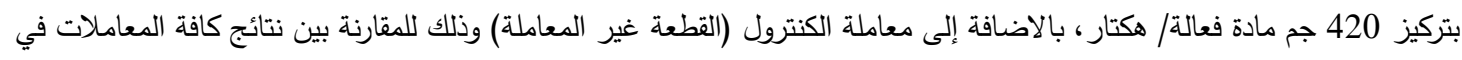

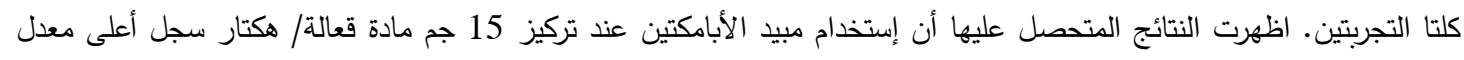

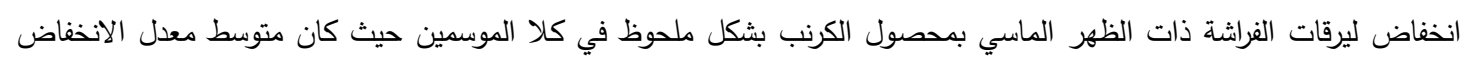

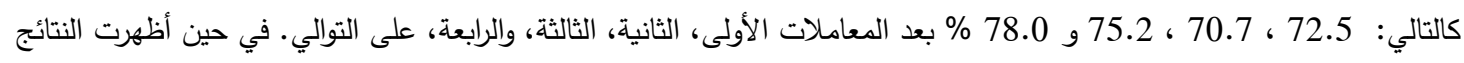

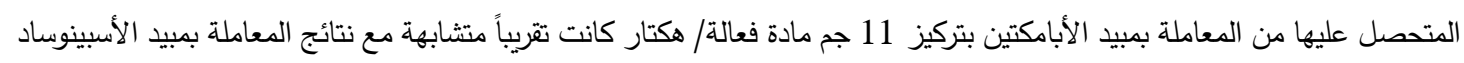

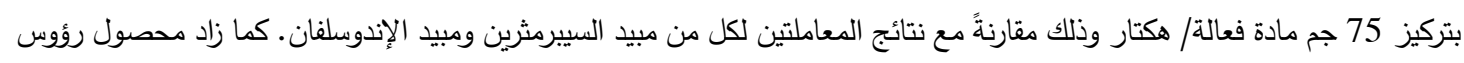

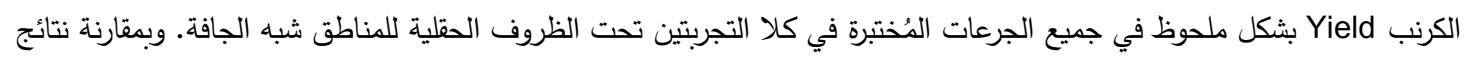

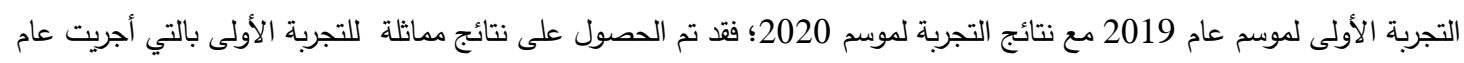

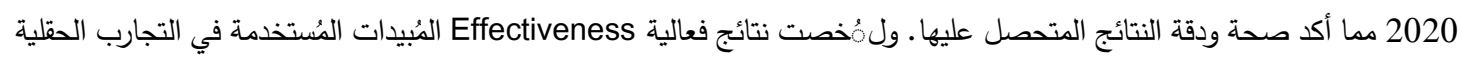

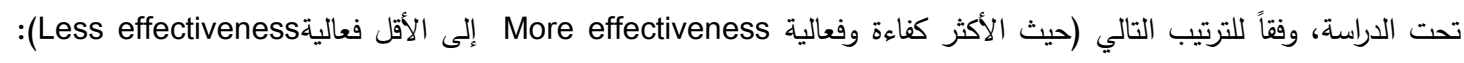

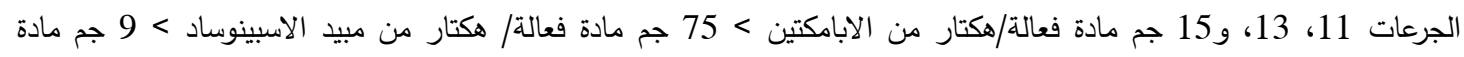

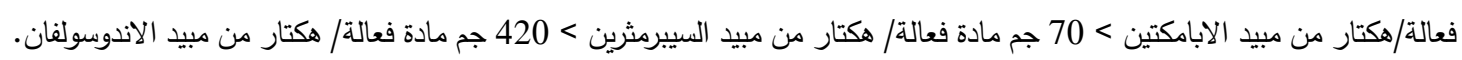

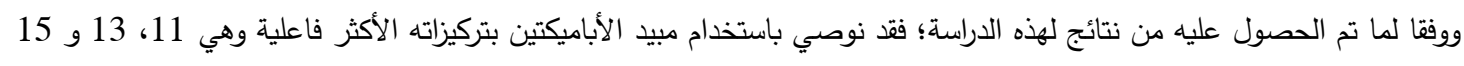

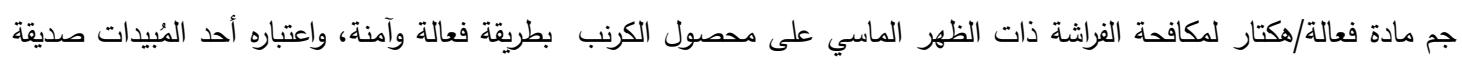

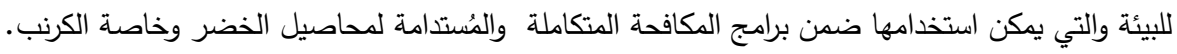

